\title{
Vehicle Routing Problem with External Carrier
}

\author{
Jan PELIKÁN \\ University of Economics, Prague, Czech Republic \\ pelikan@vse.cz
}

\begin{abstract}
The Vehicle routing problem with private (internal) and common (external) carrier is a modification of classic logistic problem. The following contribution studies this problem, assuming that primary carrier secures transport to customers by utilizing its own vehicles, whose capacities and costs per $1 \mathrm{~km}$ of transport are given and is the same for all vehicles. Also, there is given the matrix of the shortest distances between nodes (depot and customers). On the other hand, external carrier's costs depend only on transported quantity measured by weight of goods or by the number of containers/pallets. External carrier's costs do not depend on the vehicle's type, traveled distance etc. A mathematical model designed in this article is reformulated to a VRP model, where the routes may not contain all nodes. As following, there is a description of the original heuristics, which can be modified differently and three of those modifications are suggested. The results of numerical experiments for tasks of various size are presented at the end of the article. There are results of three heuristic modifications as well as results of the model solution, which due to NP difficulty and size of tasks could not be completed (the calculation was interrupted or collapsed after some time).
\end{abstract}

Keywords: Vehicle Routing Problem, Heuristics, Integer Programming.

\section{Introduction}

Many companies use services of logistics companies to transport goods to customers as an addition to transport by their own vehicles. In that case, transport is provided by internal (primary, private) carrier as well as by external (common) carrier. Primary carrier uses its own vehicles and costs are derived from traveled kilometers, i.e. the length of the vehicle's route. As for an external carrier, which secures a transport of goods for different subjects, costs are calculated differently. Mostly, it depends only on the volume of transported goods, which is measured by weight, or by the number of pallets/containers needed for transport. This dependence also may be non-linear, and the price of the transported unit may depend on the total volume of goods alternatively on the distance between the customer and the depot.

This article studies a problem with optimization of total costs consisted of external and internal carrier costs (vehicle routing problem with private and common carrier VRPPC). Demand of customers, i.e. quantity of goods which has to be transported 
from depot to customers, is given as well as costs matrix for transport between nodes (customers and depot, further graph nodes).

Suppose an internal carrier's homogeneous rolling stock with a constant cost per 1 $\mathrm{km}$ of vehicle's ride. For an external carrier, cost per unit of goods is constant and freight cost will be linearly dependent on quantity.

The problem is NP hard because classical vehicle routing problem can be reduced to this problem. Tabu search heuristic is published in [2] for the problem with nonsplit demand. A heuristic proposed in [4] selects customers according to the distance from the depot, customers who are close to the depot are assigned to the private carrier, the others to common carriers. A modification of the Clarke and Wrights savings algorithm is used in [1] to solve so-called truckload and less-than-truckload problem, where private carrier uses less-than-truckload vehicles while an outside carrier uses trucks.

A modified insert heuristic for VRPPC is presented in [3]. Routes of private vehicle is gradually created by inserting node in a route. Node has to meet condition, that the private carrier's costs increase is lower than the common carrier's costs for the transport goods to this node.

\section{$2 \quad$ Mathematical model}

VRPPC with non-split demand in nodes can be formulated as integer programming problem. Let $G=\{V ; E\}$ be undirected complete graph, $V=\{1,2, \ldots, \mathrm{n}\}$, node 1 is the depot, nodes $2,3, \ldots, n$ are customers.

\section{Parameters of the model are:}

- $d_{i j}$ cost of transport from the node $i$ to the node $j$,

- $q_{i}$ demand at the node $i$,

- $w$ capacity of primal carrier's vehicle,

- $c c$, costs of the transport of unit of goods by the common carrier.

\section{Variables of the model are:}

- $x_{i j}$ binary, equals 1 if the vehicle travels from the node $i$ to the node $j$,

- $z i$ binary, equals 1 if common carrier assures whole demand $q_{i}$ at the node $i$,

- $u i$ variables in anti-cyclic constraints.

\section{Mathematical model of VRPPC.}

$$
\begin{gathered}
f(x, z)=\sum_{i=1}^{n} \sum_{j=1}^{n} d_{i j} x_{i j}+c_{c} \sum_{i=1}^{n} q_{i} z_{i} \rightarrow \min \\
\sum_{i=1}^{n} x_{i j}=\sum_{i=1}^{n} x_{j i}, \quad j=1,2, \ldots, n \\
\sum_{\mathrm{i}=1}^{n} x_{i j}+z_{i}=1, i=1,2, \ldots, n \\
u_{i}+q_{j}-w\left(1-x_{i j}\right) \leq u_{j}, i=1,2, \ldots, n, j=2,3, \ldots, n, i \neq j
\end{gathered}
$$




$$
\begin{gathered}
u_{j} \leq w, j=1,2, \ldots, n \\
x_{i j,} \quad z_{i}, \quad \text { binary, } i, j=1,2, \ldots, n, \quad i \neq j
\end{gathered}
$$

The objective function (1) minimizes the sum of travel costs of private carrier's vehicles and common carrier's costs. Constraint (2) states that the vehicle must enter and leave node. Equation (3) means that node which is not served by the common carrier needs to be served by private carrier's vehicle. Anti-cyclic conditions and defining load $u i$ of the vehicle which is entering node $i$ are in (4). Inequality (5) assures that vehicle's capacity is not exceeded.

Theorem. Model (1)-(6) can be reformulated as vehicle routing problem with no obligation enter all nodes in the form (1'), (2), (3'), (4), (5), (6), where $d_{i j}^{\prime}=d_{i j}-$ $1 / 2 q_{i}-1 / 2 q_{j}$ and:

$$
\begin{gathered}
f^{\prime}(x)=\sum_{i=1}^{n} \sum_{j=1}^{n} d^{\prime}{ }_{i j} x_{i j} \rightarrow \min \\
\sum_{\mathrm{i}=1}^{\mathrm{n}} x_{\mathrm{ij}} \leq 1, \quad i=1,2, \ldots, n
\end{gathered}
$$

Proof. The object function (1') is:

$$
\begin{gathered}
f^{\prime}(x)=\sum_{i=1}^{n} \sum_{j=1}^{n} d^{\prime}{ }_{i j} x_{i j}= \\
\left.=\sum_{i=1}^{n} \sum_{j=1}^{n} d_{i j}-1 / 2 q_{i}-1 / 2 q_{j}\right) x_{i j}= \\
=\sum_{i=1}^{n} \sum_{j=1}^{n} d_{i j} x_{i j}-1 / 2 c_{c}\left(\sum_{i=1}^{n} q_{i} \sum_{j=1}^{n} x_{i j}+\sum_{j=1}^{n} q_{j} \sum_{i=1}^{n} x_{i j}\right)= \\
=\sum_{i=1}^{n} \sum_{j=1}^{n} d_{i j} x_{i j}-c_{c} \sum_{i=1}^{n} q_{i} \sum_{j=1}^{n} x_{i j}
\end{gathered}
$$

If we put $z_{i}=1-\sum_{\mathrm{j}=1}^{\mathrm{n}} x_{\mathrm{ij}}$ or $\sum_{\mathrm{j}=1}^{\mathrm{n}} x_{\mathrm{ij}}=1-z_{i}$, we have:

$$
\begin{gathered}
f^{\prime}(x)=\sum_{i=1}^{n} \sum_{j=1}^{n} d_{i j} x_{i j}-c_{c} \sum_{i=1}^{n} q_{i} \sum_{j=1}^{n} x_{i j}= \\
=\sum_{i=1}^{n} \sum_{j=1}^{n} d_{i j} x_{i j}+c_{c} \sum_{i=1}^{n} q_{i} z_{i}-c_{c} \sum_{i=1}^{n} q_{i}= \\
=f(x, z)-c_{c} \sum_{i=1}^{n} q_{i}=f(x, z)+\text { const. }
\end{gathered}
$$

\section{$3 \quad$ Node subset heuristic}

VRPPC with non-split demand at nodes means that the set of nodes $V$ is divided into two subsets, subset $V^{\prime}$ and subset $V$ - $V$ '. The first subset $V^{\prime}$ contains nodes which are served by the primal carrier. Common carrier transports goods from the depot to the nodes contained in the second subset $V-V$ '. In the optimal solution of VRPPC, there is an optimal subset of nodes $V^{\prime}$, and optimal routes of vehicles of the primal carrier on the subset $V^{\prime}$. If a subset of nodes $V^{\prime}$ is created, we have to optimize transport costs of the primal carrier, but costs of the common carrier for nodes from subset $\mathrm{V}-\mathrm{V}$ ' are constant.

VRPPC consists of two sub-problems:

- Finding the optimal subset of nodes V' (point a), 
- Solving the vehicle routing problem on this subset of nodes V' (point b).

Proposed heuristic consists of two heuristics: a heuristic for point a) and a heuristic for point $b$ ).

We can utilize standard heuristics (for example insert heuristic, nearest neighborhood heuristic, savings heuristic, ...) for the problem b). For the problem a) we have to choose a subset V' from all subsets of the set V. Because the number of all subsets of $\mathrm{V}$ is roughly $2^{\mathrm{n}}$, it is not possible in polynomial time to go through all possible subsets and choose the subset with the lowest costs (sum of costs of the primal and common carrier).

\subsection{The algorithm of node subset heuristic:}

Step 1. Sort the node set V by increasing value of certain criterion (it will be proposed later). The sorted node set is in the form $V=\left(v_{1}, v_{2}, \ldots, v_{n}\right)$, put $f^{*}:=$-infinity.

Step 2. Repeat for $k=2,3, \ldots, n$ : Let's take $V^{\prime}=\left\{v_{1}, v_{2}, \ldots, v_{k}\right\}$ and solve a vehicle routing problem for this subset of nodes (using a mathematical model or some heuristic), denote the value of costs $f_{1}$.

Calculate costs of external carrier according to the formula

$$
f_{2}=c_{c} \sum_{i=k+1}^{n} q_{v_{i}},
$$

if $f_{1}+f_{2}<f^{*}$, put $f^{*}:=f_{1}+f_{2}$ and $V^{*}:=V^{\prime}$.

The result of the heuristic is the value of total costs $f^{*}$, subsets of nodes $\mathrm{V}^{*}$ and the solution of the vehicle routing problem for this subset of nodes.

Sorting criterions. The following criterions can be proposed for the heuristic formulated above:

- K1: nodes are sorted by the value $d_{l i}$, that is by costs of transfer from the depot to each node.

- K2: nodes are sorted by the value of sum costs $d_{l i}$ plus $m$ values costs $d_{i j}$ for nodes $j$ for which $d_{i j}$ are lowest for $j$ different of $i$. Parameter $m$ is chosen, we can put for example $m=1,2, \ldots$.

- K3: nodes are sorted by the value $d i f_{i}=d_{I_{i}-} c_{c} q_{i}$, which is the difference between costs from the depot to node $i$ and costs of a common carrier for delivering demand $q_{i}$.

We have to solve vehicle routing problem for the subset of nodes $V^{\prime}$ in Step 2: point 1), which is NP hard problem. The problem can be solved by the mathematical model using the branch and bound method. The optimal solution will be obtained in reasonable computational time only for a small number of nodes. If the computation is interrupted after the certain time limit, we can take it as suboptimal solution. 
The second possibility is to use heuristics. For numerical experiments shown in the next chapter were used two heuristics: insert heuristic and savings heuristic.

Comment. There is a possibility to reduce the total costs of the solution if we use the following method for routes of primal carrier's vehicle. If the route $R$ of primal carrier's vehicle in the form $v_{1} \rightarrow v_{2} \rightarrow \ldots \rightarrow v_{s-1}$ meets the condition $d_{v_{k-1} v_{k}}+$ $d_{v_{k} v_{k+1}}-d_{v_{k-1} v_{k+1}}>\quad c_{c} q_{k}$ for some $k=2,3, \ldots, s-1$, then by deleting the node $v_{k}$ from route $R$ total costs decrease.

\section{$4 \quad$ Numerical experiments}

For comparing results of heuristics and the result of the mathematical model solution, examples which are published in http://www.uv.es/ belengue/carp.html were used. Results are shown in Table 1. The heuristic is written in VBA language. The model is solved using CPLEX 12.0 on PC (IntelCore2Quad, 2,83GHz).

The best values of object function, which were obtained when using mathematical model are shown in Table 1 (row "Model"), however, these results are not optimal. The gap of object function is placed under the value of total costs (in $\%$ of the lower bound). Next rows show total costs of the solution obtained by using proposed node subsets heuristics with criterion $\mathrm{K} 1, \mathrm{~K} 2$, and $\mathrm{K} 3$.

The result of the model is better than the result of heuristic in case of problems P24 and P31 which have a small number of nodes. As for the problem P77, it is the opposite.

Table 1. Results of numerical experiments.

\begin{tabular}{lcll}
\hline Total costs & P24 (24 nodes) & P31 (31 nodes) & P77 (77 nodes) \\
\hline Model & 1,200 & 2,540 & 138,020 \\
Gap & $18 \%$ & $14 \%$ & $71 \%$ \\
Computational & & & $\begin{array}{l}20 \text { minutes } \\
\text { computation } \\
\text { time }\end{array}$ \\
Was aborted \\
$K 2$ & 1,340 & 2 hours & 136,520 \\
$K 3$ & $1,270(3)^{*)}$ & 2,950 & $125,740(5)^{*)}$ \\
\hline
\end{tabular}

$\left.{ }^{*}\right) \mathrm{m}$ is number of neighboring nodes in criterion $K 2$.

\section{Conclusion}

An interesting modification for vehicle routing problem is studied, the mathematical model of the problem is presented and new heuristic method is proposed. As to solve the mathematical model for the real problem is very difficult (problem is NP hard), the proposed heuristic method provides us a good solution in polynomial time. 
Acknowledgements. Supported by the grant No. 16-00408S of the Czech Grant Agency.

\section{References}

1. Chu, C.: A heuristic algorithm for the truckload and less-than-truckload problem. European Journal of Operational Research 165(3), 657-667 (2005), 657-667 (2005), DOI: 10.1016/j.ejor.2003.08.067.

2. Côté, J., Potvin, J.: A tabu search heuristic for the vehicle routing problem with private fleet and common carrier. European Journal of Operational Research 198(2), 464-469 (2009), DOI: 10.1016/j.ejor.2008.09.009.

3. Pelikán, J.: Heuristics for VRP with private and common carriers. In: Kocourek, A., Vavroušek, M. (eds.) Mathematical Methods in Economics MME 2016, 6-9 September 2016, vol. 34, pp. 658-662, Technical University of Liberec, Liberec (2016).

4. Plevný, M.: Vehicle routing problem with a private fleet and common carriers - the variety with a possibility of sharing the satisfaction of demand. In: Vojáčková, H. (eds.) Mathematical Methods in Economics 2013, 11-13 September 2013,), vol. 31, pp.730-736. College of Polytechnics Jihlava, Jihlava (2013).

5. The Capacitated Arc Routing Problem, https://www.uv.es/belengue/carp.html, last accessed 2017-11-26. 